PHYSICAL REVIEW D 93, 039903(E) (2016)

\title{
Erratum: Soft gluon emission process in the color-octet model for heavy quarkonium production [Phys. Rev. D 60, 114025 (1999)]
}

\author{
Cheuk-Yin Wong \\ (Received 19 January 2016; published 9 February 2016)
}

DOI: 10.1103/PhysRevD.93.039903

There is a typographical error in Eq. (76) of Ref. [1] which gives the Gamow factor $K_{L}(\eta)$ for the Coulomb interaction $V(r)=\alpha / r$, for a state with an angular momentum $L$. We would like to write down the correct expression and give the derivation of the Gamow factor in Eq. (12) below.

The regular Coulomb wave function for the Coulomb potential $V(r)=\alpha / r$, as given by Eq. (14.1.3) of Ref. [2], is

$$
u_{L}(r)=F_{L}(\eta, \rho)=C_{L}(\eta) \rho^{L+1} e^{-i \rho} M(L+1-i \eta, 2 L+2,2 i \rho),
$$

where $\eta=\alpha / v$ and $\rho=k r$. We would like to represent the above wave function by the amplitude $A_{L}(r)$ and phase shift $\delta_{L}(r)$ in terms of free-potential Riccati-Bessel wave functions $\hat{j}_{L}(k r)$ and $\hat{n}_{L}(k r)$ as in Calogero [3],

$$
u_{L}(r)=\frac{A_{L}(r)}{A_{L}(\infty)}\left[\cos \delta_{L}(r) \hat{j}_{L}(k r)-\sin \delta_{L}(r) \hat{n}_{L}(k r)\right]
$$

where

$$
\begin{gathered}
\hat{j}_{L}(\rho)=\left(\frac{\pi \rho}{2}\right)^{1 / 2} J_{L+1 / 2}(\rho), \\
\hat{n}_{L}(\rho)=(-1)^{L+1}\left(\frac{\pi \rho}{2}\right)^{1 / 2} J_{-(L+1 / 2)}(\rho) .
\end{gathered}
$$

The functions $A_{L}(r)$ and $\delta_{L}(r)$ need to be solved as a function of $r$ as formulated by Calogero [1,3,4], starting with $\delta_{L}(0)=0$, to yield the proper phase shift at $r \rightarrow \infty$ appropriate for the Coulomb potential.

The Gamow factor (or $K$ factor) expresses the effects of the Coulomb interaction and is defined as the ratio of the absolute square of the amplitude at the origin and at infinity [1,4],

$$
K_{L}=\left|\frac{A_{L}(0)}{A_{L}(\infty)}\right|^{2}
$$

We can get $K_{L}$ by matching the wave functions of Eqs. (1) and (2). Near the origin with $\delta_{L}(0)=0$, Eq. (2) gives

$$
\lim _{r \rightarrow 0} u_{L}(r)=\frac{A_{L}(0)}{A_{L}(\infty)} \hat{j}_{L}(\rho)=\frac{A_{L}(0)}{A_{L}(\infty)} \frac{\rho^{L+1}}{(2 L+1) ! !} .
$$

On the other hand, in Eq. (1) as $M(r \rightarrow 0)=1$, the wave function $u_{L}$ near the origin is

$$
\lim _{r \rightarrow 0} u_{L}(r)=C_{L}(\eta) \rho^{L+1}
$$

Matching Eq. (6) with Eq. (7), we obtain

$$
\frac{A(0)}{A(\infty)}=(2 L+1) ! ! C_{L}(\eta)
$$

Therefore, the Gamow factor is related to $C_{L}(\eta)$ by 


$$
K_{L}(\eta)=\left|(2 L+1) ! ! C_{L}(\eta)\right|^{2}
$$

From Eq. (14.1.7) of [2], the Gamow factor for $L=0$ is

$$
K_{0}(\eta)=\left|C_{0}(\eta)\right|^{2}=\frac{2 \pi \eta}{\exp \{2 \pi \eta\}-1}
$$

The coefficient $C_{L}(\eta)$ satisfies the recurrence relation [2],

$$
C_{L}(\eta)=\frac{\left(L+\eta^{2}\right)^{1 / 2}}{L(2 L+1)} C_{L-1}(\eta)
$$

and thus

$$
\left|C_{L}(\eta)\right|^{2}=\frac{\left(L^{2}+\eta^{2}\right)\left[(L-1)^{2}+\eta^{2}\right] \ldots\left(1+\eta^{2}\right)}{[L !(2 L+1) ! !]^{2}} \frac{2 \pi \eta}{\exp \{2 \pi \eta\}-1}
$$

The Gamow factor for the Coulomb interaction $V(r)=\alpha / r$, for a state with angular momentum $L \geq 1$, is

$$
K_{L}(\eta)=\frac{\left(L^{2}+\eta^{2}\right)\left[(L-1)^{2}+\eta^{2}\right] \ldots\left(1+\eta^{2}\right)}{[L !]^{2}} \frac{2 \pi \eta}{\exp \{2 \pi \eta\}-1}
$$

[1] C. Y. Wong, Phys. Rev. D 60, 114025 (1999).

[2] M. Abramowitz and I. A. Stegun, Handbook of Mathematical Functions (Dover, New York, 1965).

[3] F. Calogero, Variable Phase Approach to Potential Scattering, (Academic, New York, 1967).

[4] C. Y. Wong and L. Chatterjee, Z. Phys. C 75, 523 (1997). 